\title{
A Geometric Characterization of Certain First Integrals for Nonholonomic Systems with Symmetries
}

\author{
Paula BALSEIRO † and Nicola SANSONETTO $\ddagger$ \\ $\dagger$ Universidade Federal Fluminense, Instituto de Matemática, \\ Rua Mario Santos Braga S/N, 24020-140, Niteroi, Rio de Janeiro, Brazil \\ E-mail: pbalseiro@vm.uff.br \\ $¥$ Università degli Studi di Padova, Dipartimento di Matematica, \\ via Trieste 64, 35121 Padova, Italy \\ E-mail: nicola.sansonetto@gmail.com
}

Received October 29, 2015, in final form February 12, 2016; Published online February 21, 2016 http://dx.doi.org/10.3842/SIGMA.2016.018

\begin{abstract}
We study the existence of first integrals in nonholonomic systems with symmetry. First we define the concept of $\mathcal{M}$-cotangent lift of a vector field on a manifold $Q$ in order to unify the works [Balseiro P., Arch. Ration. Mech. Anal. 214 (2014), 453-501, arXiv:1301.1091], [Fassò F., Ramos A., Sansonetto N., Regul. Chaotic Dyn. 12 (2007), 579588], and [Fassò F., Giacobbe A., Sansonetto N., Rep. Math. Phys. 62 (2008), 345-367]. Second, we study gauge symmetries and gauge momenta, in the cases in which there are the symmetries that satisfy the so-called vertical symmetry condition. Under such condition we can predict the number of linearly independent first integrals (that are gauge momenta). We illustrate the theory with two examples.
\end{abstract}

Key words: nonholonomic systems; Lie group symmetries; first integrals; gauge symmetries and gauge momenta

2010 Mathematics Subject Classification: 70F25; 70H33; 53D20

Dedicated to Sergio Benenti on the occasion of his 70th birthday

\section{Introduction}

It is well known that for Hamiltonian systems with a group of symmetries the components of the momentum map are first integrals of the system. For nonholonomic systems the situation is different: symmetries do not necessarily give rise to first integrals. Nevertheless the search for first integrals is a central topic in the study of nonholonomic systems, and different ideas to link them to the presence of symmetries have been proposed and investigated $[5,9,10,11$, $13,15,18,19,20,21,22,24,26,27,33,35]$. In this note we give a geometric interpretation of the notions of gauge symmetries ${ }^{1}$ and gauge momenta introduced in [5] and further developed in $[12,14,18,19,20]$, inspired by the tools used in [1] to study the Hamiltonization problem of nonholonomic systems.

We start by considering a $G$-invariant nonholonomic system on a manifold $Q$ given by a Lagrangian $L: T Q \rightarrow \mathbb{R}$ and a constraint distribution $D$; we let $\mathcal{M}:=\operatorname{Leg}(D)$ be the constraint submanifold of $T^{*} Q$, where Leg is the Legendre transformation (see, e.g., [7, 8, 17, 27, 30, 32]).

\footnotetext{
${ }^{\star}$ This paper is a contribution to the Special Issue on Analytical Mechanics and Differential Geometry in honour of Sergio Benenti. The full collection is available at http://www.emis.de/journals/SIGMA/Benenti.html

${ }^{1}$ It is worth noting that in this paper - as well as in $[18,19,20]$ - the term gauge symmetry is related to the search of first integrals induced by the action of a Lie group and this concept has no connection with the term gauge transformation used in [1] to "deform" brackets.
} 
For each section $\xi$ of the Lie algebra bundle $\mathfrak{g} \times Q \rightarrow Q$ we consider the function $\mathcal{J}_{\xi}: \mathcal{M} \rightarrow \mathbb{R}$ given by $\mathcal{J}_{\xi}:=\mathbf{i}_{\xi_{\mathcal{M}}} \Theta_{\mathcal{M}}$, where $\Theta_{\mathcal{M}}$ is the canonical 1-form on $T^{*} Q$ restricted to the submanifold $\mathcal{M}$ and $\xi_{\mathcal{M}}$ the infinitesimal generator of $\xi$ on $\mathcal{M}$. We call $\xi \in \mathfrak{g} \times Q$ a gauge symmetry and $\mathcal{J}_{\xi}$ a gauge momentum if the cotangent lift $\xi_{Q}^{T^{*} Q}$ of the infinitesimal generator $\xi_{Q}$ of $\xi$ leaves the Hamiltonian $H$ invariant on $\mathcal{M}$. If $\xi_{Q}$ is also a section of the constraint distribution then $\mathcal{J}_{\xi}$ is a first integral of the nonholonomic dynamics, called horizontal gauge momentum and $\xi$ is the horizontal gauge symmetry that generates it.

In this paper, we introduce the notion of $\mathcal{M}$-cotangent lift of a vector field on $Q$ and show that searching for a horizontal gauge symmetry amounts to looking for a section $\xi$ of $\mathfrak{g} \times Q \rightarrow Q$ such that the $\mathcal{M}$-cotangent lift of its infinitesimal generator $\xi_{Q}$ leaves the hamiltonian function invariant. Following [1], we observe that, upon suitable assumptions, the choice of a vertical complement $W$ of the constraint distribution $D$ induces a splitting of $\mathfrak{g} \times Q \rightarrow Q$ in such a way that horizontal gauge symmetries $\xi$ are geometrically characterized as projections of constant sections. This geometric description of horizontal gauge symmetries is alternative to the differential equations considered in [5, 18]. Moreover, if $k$ is the rank of the distribution given by the intersection of $D$ with the vertical space (the tangent space to the $G$-orbits), then we show that there are $k$ linearly independent horizontal gauge momenta.

The paper is organized as follows. In Section 2 we recall some facts about nonholonomic systems with symmetry and gauge momenta. In Section 3, we characterize horizontal gauge symmetries with respect to the $\mathcal{M}$-cotangent lift and then, using a vertical complement of the constraints, we describe the horizontal gauge momenta in terms of Lie algebra elements. Section 4 illustrates the theory with two examples. We finish with a section of conclusions and perspectives.

Throughout the paper all manifolds, distributions and maps are smooth; the distributions are assumed to be regular and the group actions are free and proper.

\section{Preliminaries: conserved quantities in nonholonomic mechanics}

\subsection{Nonholonomic systems with symmetry}

Nonholonomic systems. Let us denote by $(Q, L, D)$ a nonholonomic system on a manifold $Q$ defined by a Lagrangian $L: T Q \rightarrow \mathbb{R}$ of mechanical type and a (non-integrable) distribution $D$ on $Q$ describing the permitted velocities. The constraint submanifold $\mathcal{M} \subset T^{*} Q$ is defined by $\mathcal{M}:=\kappa^{b}(D)$, where $\kappa$ is the kinetic energy metric that induces the isomorphism $\kappa^{b}: T Q \rightarrow T^{*} Q$ defined by $\kappa^{b}(X)(Y)=\kappa(X, Y)$ for $X, Y \in T Q$. Note that since $\kappa$ is linear on the fibers, then $\mathcal{M}$ is a vector subbundle of $T^{*} Q$; we denote by $\tau_{\mathcal{M}}: \mathcal{M} \rightarrow Q$ the canonical projection. If $\mathcal{H}: T^{*} Q \rightarrow$ $\mathbb{R}$ is the Hamiltonian function associated to $L$, then we denote by $\mathcal{H}_{\mathcal{M}}: \mathcal{M} \rightarrow R$ the pull-back of the Hamiltonian $\mathcal{H}$ to $\mathcal{M}$, i.e., $\mathcal{H}_{\mathcal{M}}=\iota^{*} \mathcal{H}$, where $\iota: \mathcal{M} \rightarrow T^{*} Q$ is the natural inclusion. Moreover, we define $\Omega_{\mathcal{M}}$ the 2 -form on $\mathcal{M}$ given by $\Omega_{\mathcal{M}}:=\iota^{*} \Omega_{Q}$, where $\Omega_{Q}$ is the canonical 2 -form on $T^{*} Q$. Let $\mathcal{C}$ be the non-integrable distribution on $\mathcal{M}$ given, at each $m \in \mathcal{M}$, by

$$
\mathcal{C}_{m}:=\left\{v_{m} \in T_{m} \mathcal{M}: T \tau_{\mathcal{M}}\left(v_{m}\right) \in D_{\tau_{\mathcal{M}}(m)}\right\} .
$$

The nonholonomic dynamics is described by the integral curves of the vector field $X_{\mathrm{nh}}$ on $\mathcal{M}$ defined by

$$
\left.\mathbf{i}_{X_{\mathrm{nh}}} \Omega_{\mathcal{M}}\right|_{\mathcal{C}}=\left.d \mathcal{H}_{\mathcal{M}}\right|_{\mathcal{C}},
$$

where $\left.\Omega_{\mathcal{M}}\right|_{\mathcal{C}}$ denotes the restriction of $\Omega_{\mathcal{M}}$ to $\mathcal{C}$. Since the vector field $X_{\text {nh }}$ takes values on $\mathcal{C}$, we say that it is a section of the bundle $\mathcal{C} \rightarrow \mathcal{M}$, i.e., $X_{\mathrm{nh}} \in \Gamma(\mathcal{C})$. It is important to note that the 
solution $X_{\mathrm{nh}}$ satisfying (2.1) is unique since the 2 -section $\left.\Omega_{\mathcal{M}}\right|_{\mathcal{C}}$ is nondegenerate [6]. The vector field $X_{\mathrm{nh}}$ is also called the nonholonomic dynamics or the nonholonomic vector field.

In this setting, a function $f$ on $\mathcal{M}$ is a first integral of $X_{\mathrm{nh}}$ (or $f$ is conserved by $X_{\mathrm{nh}}$ ) if and only if $X_{\mathrm{nh}}(f)=0$. From (2.1) $f \in C^{\infty}(\mathcal{M})$ is a first integral if and only if $X_{f}\left(\mathcal{H}_{\mathcal{M}}\right)=0$, where $X_{f} \in \mathfrak{X}(\mathcal{M})$ is the nonholonomic Hamiltonian vector field ${ }^{2}$ on $\mathcal{M}$ defined by

$$
\left.\mathbf{i}_{X_{f}} \Omega_{\mathcal{M}}\right|_{\mathcal{C}}=\left.d f\right|_{\mathcal{C}}
$$

Observe that the nonholonomic Hamiltonian vector field $X_{f}$ given in (2.2) is a section of $\mathcal{C} \rightarrow \mathcal{M}$, i.e., $X_{f} \in \Gamma(\mathcal{C})$. Moreover, since $\left.\Omega_{\mathcal{M}}\right|_{\mathcal{C}}$ is nondegenerate, equation (2.2) induces an almost Poisson bracket $\{\cdot, \cdot\}_{\mathrm{nh}}$ on $\mathcal{M}$ called the nonholonomic bracket [25, 28, 34]. In fact, for every $f, g \in C^{\infty}(\mathcal{M})$ the nonholonomic bracket $\{\cdot, \cdot\}_{\mathrm{nh}}$ is defined by $\{f, g\}_{\mathrm{nh}}=-X_{f}(g)$, where $X_{f}$ is the unique vector field on $\mathcal{M}$ (with values in $\mathcal{C}$ ) that satisfies $(2.2)$. Hence, $f \in C^{\infty}(\mathcal{M})$ is a first integral of the nonholonomic dynamics if and only if $\left\{f, \mathcal{H}_{\mathcal{M}}\right\}_{\mathrm{nh}}=0$, in which case we say that the functions $f$ and $\mathcal{H}_{\mathcal{M}}$ are in involution.

As it is well known there is a correspondence between almost Poisson brackets $\{\cdot, \cdot\}$ on a manifold $\mathcal{M}$ and bivector fields $\pi \in \Gamma\left(\Lambda^{2}(T \mathcal{M})\right)$, defined by $\pi(d f, d g)=\{f, g\}$, for $f, g \in C^{\infty}(\mathcal{M})$ (see, e.g., [29]). Throughout the paper we will work indistinguishably with almost Poisson brackets and bivector fields. We denote by $\pi^{\sharp}: T^{*} \mathcal{M} \longrightarrow T \mathcal{M}$ the map such that $\beta\left(\pi^{\sharp}(\alpha)\right)=$ $\pi(\alpha, \beta)$. In our context, the nonholonomic bracket $\{\cdot, \cdot\}_{\mathrm{nh}}$ induces the nonholonomic bivector field $\pi_{\mathrm{nh}}$ and the nonholonomic dynamics is given by the vector field $X_{\mathrm{nh}}=-\pi_{\mathrm{nh}}^{\sharp}\left(d \mathcal{H}_{\mathcal{M}}\right)$.

Symmetries. Let $G$ be a Lie group acting on $Q$ freely and properly. We say that the $G$-action is a symmetry of the nonholonomic system $(Q, L, D)$ if the tangent lift of the action to $T Q$ leaves $L$ and $D$ invariant. In this case, the cotangent lift of the $G$-action to $T^{*} Q$ leaves the constraint submanifold $\mathcal{M}$ invariant and thus it induces, by restriction, a $G$-action on $\mathcal{M}$ :

$$
\phi: \quad G \times \mathcal{M} \rightarrow \mathcal{M} .
$$

It is straightforward to see that the Hamiltonian $\mathcal{H}_{\mathcal{M}}$ and the nonholonomic bracket $\{\cdot, \cdot\}_{\mathrm{nh}}$ are $G$-invariant [25, 28].

Let us denote by $V$ the distribution on $Q$ whose fibers $V_{q}$, for $q \in Q$, are the tangent spaces to the orbits of $G$ in $Q$, that is $V_{q}=T\left(\operatorname{Orb}_{q}(q)\right)$. Equivalently, $\mathcal{V}$ is the distribution on $\mathcal{M}$ such that for each $m \in \mathcal{M}$ the fiber $\mathcal{V}_{m}$ is the tangent space to the orbit of $G$ in $\mathcal{M}$. Since the action on $\mathcal{M}$ is lifted from an action on $Q$ then, for each $m \in \mathcal{M}$, the fibers $\mathcal{V}_{m}$ and $V_{\tau_{\mathcal{M}}(m)}$ are diffeomorphic. We call $V$ and $\mathcal{V}$ the vertical spaces associated to the $G$-action on $Q$ and on $\mathcal{M}$, respectively. For each $\eta \in \mathfrak{g}$, where $\mathfrak{g}$ denotes the Lie algebra of $G, \eta_{Q}$ and $\eta_{T^{*} Q}$ denote the infinitesimal generator associated to the $G$-action on $Q$ and on $T^{*} Q$, respectively. Since $\mathcal{M}$ is an invariant submanifold of $T^{*} Q$, for each $\eta \in \mathfrak{g}$ the infinitesimal generator $\eta_{\mathcal{M}}$ of $\eta$ on $\mathcal{M}$ is well defined; $\eta_{T^{*} Q}(m)=\eta_{\mathcal{M}}(m) \in T_{m} \mathcal{M}$, for each $m \in \mathcal{M}$.

Consider the distributions $S$ and $\mathcal{S}$ on $Q$ and $\mathcal{M}$, respectively, given by

$$
S_{q}=D_{q} \cap V_{q} \quad \text { and } \quad \mathcal{S}_{m}=\mathcal{C}_{m} \cap \mathcal{V}_{m},
$$

for $q \in Q$ and $m \in \mathcal{M}$. The distribution $S$ induces a vector subbundle $\mathfrak{g}_{S} \rightarrow Q$ of the trivial vector bundle $\mathfrak{g} \times Q \rightarrow Q$, defined by

$$
\mathfrak{g}_{S}=\left\{\xi_{q} \in \mathfrak{g} \times Q: \xi_{Q}(q) \in S_{q}\right\},
$$

where $\xi_{Q}(q):=\left(\xi_{q}\right)_{Q}(q)$. Observe that, for $\xi \in \Gamma(\mathfrak{g} \times Q)$, the corresponding infinitesimal generator $\xi_{\mathcal{M}}$ of the lifted action on $\mathcal{M}$ is defined by $\xi_{\mathcal{M}}(m):=\left(\xi_{q}\right)_{\mathcal{M}}(m) \in \Gamma(\mathcal{V})$ having in mind that, for a fixed $q \in Q, \xi_{q} \in \mathfrak{g}$.

\footnotetext{
${ }^{2} X_{f}$ is also called distributional Hamiltonian vector field, see [17].
} 
The nonholonomic momentum map [9] $\mathcal{J}^{\mathrm{nh}}: \mathcal{M} \rightarrow \mathfrak{g}_{S}^{*}$ is given ${ }^{3}$, at each $m \in \mathcal{M}$ and $\xi \in \Gamma\left(\mathfrak{g}_{S}\right)$, by

$$
\left\langle\mathcal{J}^{\mathrm{nh}}(m), \xi(m)\right\rangle:=\mathbf{i}_{\xi_{\mathcal{M}}} \Theta_{\mathcal{M}}(m)
$$

where $\Theta_{\mathcal{M}}$ is the pull-back to $\mathcal{M}$ of the canonical 1-form $\Theta_{Q}$ on $T^{*} Q$ via the natural inclusion $\iota$, i.e., $\Theta_{\mathcal{M}}:=\iota^{*} \Theta_{Q}$, see $[1,9]$.

Remark 2.1. The nonholonomic momentum map can be evaluated at non-constant sections of the bundle $\mathfrak{g} \times \mathcal{M} \rightarrow \mathcal{M}$. If there is a constant section $\eta \in \mathfrak{g}$ such that $\eta \in \Gamma\left(\mathfrak{g}_{S}\right)$, then $\left\langle\mathcal{J}^{\text {nh }}, \eta\right\rangle=\langle\mathcal{J}, \eta\rangle$, where $\mathcal{J}: \mathcal{M} \rightarrow \mathfrak{g}^{*}$ is the restriction to $\mathcal{M}$ of the canonical momentum map defined on $T^{*} Q$.

As it is already well known $[5,14,18]$, the nonholonomic momentum map gives a candidate for a first integral of a nonholonomic system with symmetry. In fact, by $(2.4)$, for $\xi \in \Gamma\left(\mathfrak{g}_{S}\right)$,

$$
X_{\mathrm{nh}}\left(\left\langle\mathcal{J}^{\mathrm{nh}}, \xi\right\rangle\right)=£_{\xi_{\mathcal{M}}} \Theta_{\mathcal{M}}\left(X_{\mathrm{nh}}\right)+\Omega_{\mathcal{M}}\left(\xi_{\mathcal{M}}, X_{\mathrm{nh}}\right) .
$$

Since $\xi_{\mathcal{M}} \in \Gamma(\mathcal{S})$ then $\Omega_{\mathcal{M}}\left(\xi_{\mathcal{M}}, X_{\text {nh }}\right)=-d \mathcal{H}_{\mathcal{M}}\left(\xi_{\mathcal{M}}\right)=0$, where in the last equality we use the $G$-invariance of the Hamiltonian $\mathcal{H}_{\mathcal{M}}$. We conclude that for a section $\xi \in \Gamma\left(\mathfrak{g}_{S}\right)$, the function $\left\langle\mathcal{J}^{\mathrm{nh}}, \xi\right\rangle$ is constant along the flow of the nonholonomic dynamics if and only if $\left(£_{\xi_{\mathcal{M}}} \Theta_{\mathcal{M}}\right)\left(X_{\mathrm{nh}}\right)=0$.

\subsection{Conserved quantities and cotangent lifts}

Given a vector field $X$ on $Q$, we denote by $X^{T^{*} Q}$ the cotangent lift of $X$ to $T^{*} Q$. Recall that the cotangent lift of $X$ is the infinitesimal generator of $T \Phi_{t}^{X}: T^{*} Q \rightarrow T^{*} Q$, where $T \Phi_{t}^{X}$ is the lift to $T^{*} Q$ of the flow $\Phi_{t}^{X}$ of $X$ with respect to the canonical projection $\tau_{Q}: T^{*} Q \longrightarrow Q$. In other words, the cotangent lift of $X$ can be expressed as a Hamiltonian vector field with respect to the canonical symplectic structure on $T^{*} Q$. That is, given $X \in \mathfrak{X}(Q)$ we denote by $\tilde{X}$ the linear function on $T^{*} Q$ given by $\tilde{X}(\alpha)=\alpha(X)$ for $\alpha \in T^{*} Q$, then $X^{T^{*} Q}=-\pi_{Q}^{\sharp}(d \tilde{X})$, where $\pi_{Q}$ is the bivector field associated to the canonical symplectic form $\Omega_{Q}$. In local coordinates, if $\left\{X_{i}\right\}$ is a local basis of $T Q$ and $\left(q, p_{i}\right)$ denote the local coordinates in $T^{*} Q$ associated to the dual basis $\left\{X^{i}\right\}$, then $X_{i}^{T^{*} Q}=-\pi_{Q}^{\sharp}\left(d p_{i}\right)=X_{i}+p_{k} C_{i j}^{k} \partial_{p_{j}}$, where $C_{i j}^{k} \in C^{\infty}(Q)$ are the structure functions $\left[X_{i}, X_{j}\right]=C_{i j}^{k} X_{k}$. If $X=f_{i} X_{i}$ for $f_{i} \in C^{\infty}(Q)$, then

$$
X^{T^{*} Q}=f_{i}^{v} X_{i}^{T^{*} Q}-p_{i} \pi_{Q}^{\sharp}\left(d f_{i}\right)=f_{i}^{v} X_{i}^{T^{*} Q}-p_{i} X_{j}\left(f_{i}\right) \partial_{p_{j}},
$$

where $f_{i}^{v}=\tau_{Q}^{*} f$ for $\tau_{Q}: T^{*} Q \rightarrow Q$, see [29].

In this paper, we will focus on the cotangent lift $\xi_{Q}^{T^{*} Q}$ of infinitesimal generators of sections $\xi$ of $\Gamma(\mathfrak{g} \times Q)$. It is important to note that if $\eta \in \mathfrak{g}$ (i.e., $\eta$ is a constant section of $\mathfrak{g} \times Q \rightarrow Q$ ) then $\eta_{Q}^{T^{*} Q}=\eta_{T^{*} Q}$. However this is not necessarily true if we consider non-constant sections $\xi \in \Gamma(\mathfrak{g} \times Q)$, indeed the lift of a section in $\Gamma(V)$ might not be tangent to the orbits of the lifted action to $T^{*} Q$. On the other hand, for a section $\xi \in \Gamma(\mathfrak{g} \times Q)$ we have that the corresponding linear function of $\xi_{Q}$ is given by $\tilde{\xi_{Q}}=\mathbf{i}_{\xi_{T^{*}}} \Theta_{Q}=\langle J, \xi\rangle$ and thus

$$
\xi_{Q}^{T^{*} Q}=-\pi_{Q}^{\sharp}(d\langle J, \xi\rangle) .
$$

Following $[18,19]$ we say that a gauge symmetry of a nonholonomic system $(Q, L, D)$ with symmetry $G$, is a section $\xi$ of $\mathfrak{g} \times Q \rightarrow Q$ such that the cotangent lift $\xi_{Q}^{T^{*} Q}$ of the infinitesimal

\footnotetext{
${ }^{3} \mathfrak{g}_{S}^{*}$ denotes the dual vector bundle of $\mathfrak{g}_{S}$.
} 
generator $\xi_{Q}$ leaves the Hamiltonian $\mathcal{H}$ invariant on $\mathcal{M}$, i.e., $\xi_{Q}^{T^{*} Q}(\mathcal{H})(m)=0, \forall m \in \mathcal{M}$. The gauge momentum $\mathcal{J}_{\xi}: \mathcal{M} \rightarrow \mathbb{R}$ of a gauge symmetry $\xi$ is the function

$$
\mathcal{J}_{\xi}:=\iota^{*}\left(i_{\xi_{Q}^{T^{*} Q}} \Theta_{Q}\right) .
$$

Next proposition clarifies the link between gauge momenta and first integrals

Proposition 2.2 ([21]). Consider a nonholonomic system $(Q, L, D)$ with symmetry $G$ and a section $\xi \in \Gamma\left(\mathfrak{g}_{S}\right)$. Then $\xi_{Q}^{T^{*} Q}(\mathcal{H})(m)=0, \forall m \in \mathcal{M}$, if and only if $\mathcal{J}_{\xi}$ is a first integral of the nonholonomic dynamics $X_{\mathrm{nh}}$.

When a gauge symmetry $\xi$ is also a section of $\mathfrak{g}_{S}$ we say that $\xi$ and, with a slight abuse, $\xi_{Q}$ are $D$-gauge symmetries or horizontal gauge symmetries. In this case, the first integral $\mathcal{J}_{\xi}$ is a D-gauge momentum or an horizontal gauge momentum. If $\xi \in \Gamma\left(\mathfrak{g}_{S}\right)$ is a constant section (i.e., $\xi \in \mathfrak{g}$ such that $\left.\xi_{Q} \in \Gamma(D)\right)$, then $\xi$ is called a D-symmetry or an horizontal symmetry [9, 18].

\section{Remarks 2.3.}

(i) Observe that the nonholonomic momentum (2.4) and the gauge momentum (2.7) coincide. In fact, for $\xi \in \Gamma\left(\mathfrak{g}_{S}\right)$ and for all $m \in \mathcal{M}$,

$$
\begin{aligned}
\left\langle\mathcal{J}^{\mathrm{nh}}, \xi\right\rangle(m) & =\mathbf{i}_{\xi_{\mathcal{M}}} \Theta_{\mathcal{M}}(m)=\left\langle\iota^{*} m, \xi_{Q}\right\rangle=\left\langle\iota^{*} m, T \tau_{Q}\left(\xi_{Q}^{T^{*} Q}\right)\right\rangle \\
& =\iota^{*}\left(\mathbf{i}_{\xi_{Q}^{T^{*}}} \Theta_{Q}\right)(m)=\mathcal{J}_{\xi}(m) .
\end{aligned}
$$

(ii) In [20] the definition of gauge momenta is given without the hypothesis of invariance of the Hamiltonian. However, in this paper the cases of interest are the ones in which the Hamiltonian is invariant. For a discussion of this see [20].

\section{Gauge-symmetries and the relation with a vertical complement of the constraints}

\subsection{The $\mathcal{M}$-cotangent lift}

In this section, we study horizontal gauge symmetries in terms of vector fields and functions on $\mathcal{M}$, in order to unify the viewpoints of Sections 2.1 and 2.2 .

Following $[6,17]$ we recall that, for all $m \in \mathcal{M}$

$$
T_{m}\left(T^{*} Q\right)=\mathcal{C}_{m} \oplus \mathcal{C}_{m}^{\Omega}
$$

where $\mathcal{C}_{m}^{\Omega}:=\left\{v_{m} \in T_{m}\left(T^{*} Q\right): \Omega_{Q}\left(v_{m}, w_{m}\right)=0 \forall w_{m} \in \mathcal{C}_{m}\right\}$ is the symplectic orthogonal to $\mathcal{C}_{m}$. If we denote by $\mathcal{T}_{\mathcal{C}}(m): T_{m}\left(T^{*} Q\right) \rightarrow \mathcal{C}_{m}$ the projection associated to decomposition (3.1), then to every $X \in \mathfrak{X}\left(T^{*} Q\right)$ we can associate a vector field on $\mathcal{M}$ with value on $\mathcal{C}$ by $\mathcal{T}_{\mathcal{C}}(X) \in \Gamma(\mathcal{C})$. Moreover, for each $m \in \mathcal{M}$,

$$
\pi_{\mathrm{nh}}^{\sharp}(d f)(m)=\mathcal{T}_{\mathcal{C}}\left(\pi_{Q}^{\sharp}(d \tilde{f})\right)(m),
$$

where $f \in C^{\infty}(\mathcal{M})$ and $\tilde{f} \in C^{\infty}\left(T^{*} Q\right)$ is an extension of $f$, i.e., $\left.\tilde{f}\right|_{\mathcal{M}}=f$. Observe that (3.2) is independent from the choice of the extension, see [25, 33].

Definition 3.1. For a section $\xi \in \Gamma(\mathfrak{g} \times Q)$, the $\mathcal{M}$-cotangent lift of $\xi_{Q} \in \mathfrak{X}(Q)$ is the vector field $\xi_{Q}^{\mathcal{M}}$ on $\mathcal{M}$ with values on $\mathcal{C}$ defined, at each $m \in \mathcal{M}$, by

$$
\xi_{Q}^{\mathcal{M}}(m):=\mathcal{T}_{\mathcal{C}}\left(\xi_{Q}^{T^{*} Q}\right)(m)
$$


Lemma 3.2. If $\xi \in \Gamma\left(\mathfrak{g}_{S}\right)$ is a section given by $\xi=f_{i} \eta_{i},{ }^{4}$ where $f_{i} \in C^{\infty}(Q)$ and $\eta_{i} \in \mathfrak{g}$ then,

(i) $\xi_{Q}^{\mathcal{M}}=\xi_{\mathcal{M}}+\left\langle\mathcal{J}, \eta_{i}\right\rangle \pi_{\mathrm{nh}}^{\sharp}\left(d f_{i}\right)$ where, in this case, $f_{i}$ represents the pull back of the functions $f_{i}$ to $\mathcal{M}$;

(ii) $\xi_{Q}^{\mathcal{M}}=-\pi_{\mathrm{nh}}^{\sharp}\left(d\left\langle\mathcal{J}^{\mathrm{nh}}, \xi\right\rangle\right)$.

Proof. To see $(i)$ observe that $\xi_{Q}^{\mathcal{M}}(m)=\mathcal{T}_{\mathcal{C}}\left(\xi_{T^{*} Q}\right)(m)+\left\langle J, \eta_{i}\right\rangle \mathcal{T}_{\mathcal{C}}\left(\pi_{Q}^{\sharp}\left(d f_{i}\right)\right)(m)$ where we keep the notation $f_{i}$ to denote the pull back of the functions $f_{i}$ to $T^{*} Q$. Item $(i i)$ is a consequence of (2.6) and Definition 3.1.

Next, we give another insight of Proposition 2.2 but in terms of $\mathcal{M}$-cotangent lifts and the nonholonomic momentum map and we prove it using the geometrical approach of Section 2.1.

Proposition 3.3. Given a nonholonomic system $(Q, L, D)$ and a section $\xi \in \Gamma\left(\mathfrak{g}_{S}\right)$, then

(i) $£_{\xi_{\mathcal{M}}} \Theta_{\mathcal{M}}\left(X_{\mathrm{nh}}\right)=\xi_{Q}^{\mathcal{M}}\left(\mathcal{H}_{\mathcal{M}}\right)$;

(ii) The function $\left\langle\mathcal{J}^{\mathrm{nh}}, \xi\right\rangle$ is a first integral of the nonholonomic dynamics $X_{\mathrm{nh}}$ if and only if $\xi_{Q}^{\mathcal{M}}\left(\mathcal{H}_{\mathcal{M}}\right)=0$.

Proof. To prove $(i)$, first observe that $£_{\xi_{\mathcal{M}}} \Theta_{\mathcal{M}}=f_{i} £_{\left(\eta_{i}\right)_{\mathcal{M}}} \Theta_{\mathcal{M}}+\left\langle\mathcal{J}, \eta_{i}\right\rangle d f_{i}=\left\langle\mathcal{J}, \eta_{i}\right\rangle d f_{i}$ by the $G$-invariance of $\Theta_{\mathcal{M}}$. Then we obtain that

$$
£_{\xi_{\mathcal{M}}} \Theta_{\mathcal{M}}\left(X_{\mathrm{nh}}\right)=\left\langle\mathcal{J}, \eta_{i}\right\rangle X_{\mathrm{nh}}\left(f_{i}\right)=\left\langle\mathcal{J}, \eta_{i}\right\rangle \pi_{\mathrm{nh}}^{\sharp}\left(d f_{i}\right)\left(\mathcal{H}_{\mathcal{M}}\right) .
$$

On the other hand, using Lemma 3.2(i) and since the hamiltonian $\mathcal{H}_{\mathcal{M}}$ is $G$-invariant, we have that $\xi_{Q}^{\mathcal{M}}\left(\mathcal{H}_{\mathcal{M}}\right)=\left\langle\mathcal{J}, \eta_{i}\right\rangle \pi_{\mathrm{nh}}^{\sharp}\left(d f_{i}\right)\left(\mathcal{H}_{\mathcal{M}}\right)$ and hence $£_{\xi_{\mathcal{M}}} \Theta_{\mathcal{M}}\left(X_{\mathrm{nh}}\right)=\xi_{Q}^{\mathcal{M}}\left(\mathcal{H}_{\mathcal{M}}\right)$.

Item $(i i)$ is a trivial consequence of $(i)$ since, as already seen in $(2.5)$, for $\xi \in \Gamma\left(\mathfrak{g}_{S}\right)$ the function $\mathcal{J}_{\xi}=\left\langle\mathcal{J}^{\mathrm{nh}}, \xi\right\rangle$ is conserved if and only if $£_{\xi_{\mathcal{M}}} \Theta_{\mathcal{M}}\left(X_{\mathrm{nh}}\right)=0$, which is equivalent to ask for $\xi_{Q}^{\mathcal{M}}\left(\mathcal{H}_{\mathcal{M}}\right)=0$.

Remark 3.4. Given a symplectic manifold $(P, \Omega)$, a Cartan symmetry $Z \in \mathfrak{X}(P)$ of a Hamiltonian vector field $X_{H}$ is a vector field satisfying $Z(H)=0$ and $£_{Z} \Omega=0$ [16]. In our setting, if $\xi$ is an horizontal gauge symmetry then $\xi_{\mathcal{M}}\left(\mathcal{H}_{\mathcal{M}}\right)=0$ but $£_{\xi_{\mathcal{M}}} \Omega_{\mathcal{M}}=d \alpha$, where $\alpha$ is a 1-form on $\mathcal{M}$ such that $\alpha\left(X_{\mathrm{nh}}\right)=0$. Thus $\xi_{\mathcal{M}} \in \mathfrak{X}(\mathcal{M})$ could be interpreted as a nonholonomic Cartan symmetry for the nonholonomic system $\left(\mathcal{M}, \pi_{\mathrm{nh}}, \mathcal{H}_{\mathcal{M}}\right)$. On the other hand in [15, Theorem 1] a Cartan-type symmetry of a nonholonomic systems is a vector field that leaves the Hamiltonian invariant and such that $\alpha(X)=0$ for all $X \in \Gamma(\mathcal{C})$, while we ask only for $\alpha$ to annihilate only $X_{\mathrm{nh}}$. In fact, we will see in Example 4.2 that the Chaplygin ball satisfies that $\left.\alpha\right|_{\mathcal{C}} \neq 0$.

Proposition 3.3 gives a characterization of horizontal gauge symmetries and horizontal gauge momenta in terms of the $\mathcal{M}$-cotangent lifts, so we conclude

Corollary 3.5. Consider a nonholonomic system $(Q, L, D)$ with symmetry $G$. A section $\xi \in$ $\Gamma\left(\mathfrak{g}_{S}\right)$ is a horizontal gauge symmetry if and only if the $\mathcal{M}$-cotangent lift $\xi_{Q}^{\mathcal{M}}$ of the infinitesimal generator $\xi_{Q}$ leaves the Hamiltonian $\mathcal{H}_{\mathcal{M}}$ invariant. In this case, the associated horizontal gauge momentum is the first integral $\mathcal{J}_{\xi}: \mathcal{M} \rightarrow \mathbb{R}$ given by

$$
\mathcal{J}_{\xi}:=\mathbf{i}_{\xi_{Q}^{\mathcal{M}}} \Theta_{\mathcal{M}}=\left\langle\mathcal{J}^{\text {nh }}, \xi\right\rangle .
$$

\footnotetext{
${ }^{4}$ Observe that $V$ is a regular distribution since the $G$-action is locally free [31], then each section $\xi \in \Gamma(\mathfrak{g} \times Q)$ can be written as linear combinations of elements in $\mathfrak{g}$.
} 
Proof. From Propositions 2.2 and 3.3 we see that a section $\xi \in \Gamma\left(\mathfrak{g}_{S}\right)$ satisfies that $\xi_{Q}^{T^{*} Q}(\mathcal{H})(m)$ $=0$ for all $m \in \mathcal{M}$, if and only if $\xi_{Q}^{\mathcal{M}}\left(\mathcal{H}_{\mathcal{M}}\right)=0$. Lemma 3.2 ensures that the function $\mathcal{J}_{\xi}=\mathbf{i}_{\xi_{Q}} \Theta_{\mathcal{M}}$ coincides with the definition given in (2.7) and of course with the nonholonomic momentum map (2.4). In fact, since $T \tau_{\mathcal{M}}\left(\xi_{Q}^{\mathcal{M}}\right)=\xi_{Q}$ then,

$$
\mathcal{J}_{\xi}(m)=\mathbf{i}_{\xi_{Q}^{\mathcal{M}}} \Theta_{\mathcal{M}}(m)=\left\langle\iota^{*} m, T \tau_{\mathcal{M}}\left(\xi_{Q}^{\mathcal{M}}\right)\right\rangle=\left\langle\iota^{*} m, \xi_{Q}\right\rangle=\left\langle\mathcal{J}^{\mathrm{nh}}, \xi\right\rangle(m)
$$

From Proposition 3.3 we see that the problem of finding a first integral of the nonholonomic dynamics - that is a horizontal gauge momentum - is reduced to finding a section $\xi \in \Gamma\left(\mathfrak{g}_{S}\right)$ such that $\xi_{Q}^{\mathcal{M}}\left(\mathcal{H}_{\mathcal{M}}\right)=0$. Still, the choice of such a section $\xi \in \Gamma\left(\mathfrak{g}_{S}\right)$ is not canonical. However, it was seen in [1] that, under certain circumstances, there is a "natural" choice of a section $\xi \in \Gamma\left(\mathfrak{g}_{S}\right)$ so that $\mathcal{J}_{\xi}$ is a first integral. This section $\xi$ depends on the choice of a vertical complement of the constraints. We will investigate and relate these view-points in the next section.

\subsection{Splitting adapted to the constraints and the vertical symmetry condition}

Consider a nonholonomic system $(Q, L, D)$ with a $G$-symmetry. We say that the $G$-symmetry verifies the dimension assumption if

$$
T_{q} Q=D_{q}+V_{q}, \quad \text { for } q \in Q
$$

or equivalently if

$$
T_{m} \mathcal{M}=\mathcal{C}_{m}+\mathcal{V}_{m}, \quad \text { for } m \in \mathcal{M} .
$$

Let us consider a $G$-invariant vertical complement $W$ of the constraints $D$, that is, a $G$ invariant distribution on $Q$ such that for each $q \in Q$,

$$
T_{q} Q=D_{q} \oplus W_{q}, \quad \text { and } \quad W_{q} \subset V_{q} .
$$

Let now $\mathcal{W}$ be the distribution on $\mathcal{M}$ given, at each $m \in \mathcal{M}$, by $\mathcal{W}_{m}:=\left(T \tau_{\mathcal{M}} \mid \mathcal{V}\right)^{-1}\left(W_{\tau(m)}\right)$, where $\left.T \tau_{\mathcal{M}}\right|_{\mathcal{V}}: \mathcal{V} \rightarrow V$ is the restriction of $T \tau_{\mathcal{M}}$ to $\mathcal{V} \subset T \mathcal{M}$. Then $\mathcal{W}$ is a $G$-invariant vertical complement of $\mathcal{C}$ since, for each $m \in \mathcal{M}$,

$$
T_{m} \mathcal{M}=\mathcal{C}_{m} \oplus \mathcal{W}_{m}, \quad \text { and } \quad \mathcal{W}_{m} \subset \mathcal{V}_{m}
$$

Splitting (3.4) induces a splitting of $V$ or equivalently, (3.5) induces a splitting of $\mathcal{V}$ :

$$
V_{q}=S_{q} \oplus W_{q} \quad \text { and } \quad \mathcal{V}_{m}=\mathcal{S}_{m} \oplus \mathcal{W}_{m}
$$

Therefore, the Lie algebra $\mathfrak{g}$ admits a splitting induced by (3.6) given, at each $q \in Q$, by

$$
\left.\mathfrak{g}\right|_{q}=\left.\left.\mathfrak{g}_{S}\right|_{q} \oplus \mathfrak{g}_{W}\right|_{q},
$$

where $\mathfrak{g}_{W} \rightarrow Q$ is the vector subbundle of the trivial vector bundle $\mathfrak{g} \times Q \rightarrow Q$ defined by

$$
\mathfrak{g}_{W}=\left\{\xi_{q} \in \mathfrak{g} \times Q: \xi_{Q}(q) \in W_{q}\right\} .
$$

Let us denote by $P_{\mathfrak{g}_{S}}: \mathfrak{g} \rightarrow \mathfrak{g}_{S}$ and $P_{\mathfrak{g}_{W}}: \mathfrak{g} \rightarrow \mathfrak{g}_{W}$ the projections associated to decomposition (3.7).

Remark 3.6. For every $\eta \in \mathfrak{g}$ we have that $P_{D}\left(\eta_{Q}\right)=\left(P_{\mathfrak{g}_{S}}(\eta)\right)_{Q}$ and $P_{\mathcal{C}}\left(\eta_{\mathcal{M}}\right)=\left(P_{\mathfrak{g}_{S}}(\eta)\right)_{\mathcal{M}}$, where $P_{D}: T Q \rightarrow D$ and $P_{\mathcal{C}}: T \mathcal{M} \rightarrow \mathcal{C}$ are the projections associated to the decompositions (3.4) and (3.5), respectively. 
Definition 3.7 ([1, Section 4.4]). We say that $W$ satisfies the vertical symmetry condition if $\mathfrak{g}_{W}$ is a trivial bundle over $\mathcal{M}$, that is, if $\mathfrak{g}_{W}$ is a Lie subalgebra of $\mathfrak{g}$.

When the vertical symmetry condition is satisfied there is a natural candidate to be chosen as horizontal gauge symmetry. In order to properly state next proposition, we need to introduce a 2 -form, denoted by $\left\langle\mathcal{J}, \mathcal{K}_{\mathcal{W}}\right\rangle$ (see [1]), arising from the data that defines a nonholonomic system with a $G$-symmetry satisfying the dimension assumption (3.3). More precisely, a $G$-invariant vertical complement $\mathcal{W}$ induces a $\mathfrak{g}$-valued 1 -form $\mathcal{A}_{\mathcal{W}}: T \mathcal{M} \rightarrow \mathfrak{g}$ defined by $\mathcal{A}_{\mathcal{W}}\left(v_{m}\right)=\xi$ if and only if $P_{\mathcal{W}}\left(v_{m}\right)=\xi_{\mathcal{M}}(m)$ for each $m \in \mathcal{M}$, where $P_{\mathcal{W}}: T \mathcal{M} \rightarrow \mathcal{W}$ is the projection associated to decomposition (3.5). Then, the associated $\mathcal{W}$-curvature is the $\mathfrak{g}$-valued 2 -form on $\mathcal{M}$ given by

$$
\mathcal{K}_{\mathcal{W}}(X, Y):=d \mathcal{A}_{\mathcal{W}}\left(P_{\mathcal{C}}(X), P_{\mathcal{C}}(Y)\right) \quad \text { for } X, Y \in T \mathcal{M} .
$$

Finally $\left\langle\mathcal{J}, \mathcal{K}_{\mathcal{W}}\right\rangle$ is the 2 -form on $\mathcal{M}$ defined by the natural pairing between $\mathcal{J}: \mathcal{M} \rightarrow \mathfrak{g}^{*}$ - the restriction to $\mathcal{M}$ of the canonical momentum map - and the $\mathfrak{g}$-valued 2-form $\mathcal{K}_{\mathcal{W}}$.

Proposition 3.8. Suppose that the G-invariant vertical complement $W$ satisfies the verticalsymmetry condition. If for $\eta \in \mathfrak{g}$ we have that $\left\langle\mathcal{J}, \mathcal{K}_{\mathcal{W}}\right\rangle\left(X_{\mathrm{nh}},\left(P_{\mathfrak{g}_{S}}(\eta)\right)_{\mathcal{M}}\right)=0$ then

(i) $f_{\eta}=\left\langle\mathcal{J}^{\mathrm{nh}}, P_{\mathfrak{g}_{S}}(\eta)\right\rangle$ is a first integral of the nonholonomic dynamics $X_{\mathrm{nh}}$, which is linear in the momenta.

(ii) The $\mathcal{M}$-cotangent lift $\left(P_{\mathfrak{g}_{S}}(\eta)\right)_{Q}^{\mathcal{M}} \in \mathfrak{X}(\mathcal{M})$ of $\left(P_{\mathfrak{g}_{S}}(\eta)\right)_{Q} \in \mathfrak{X}(Q)$ satisfies $\left(P_{\mathfrak{g}_{S}}(\eta)\right)_{Q}^{\mathcal{M}}\left(\mathcal{H}_{\mathcal{M}}\right)=0$.

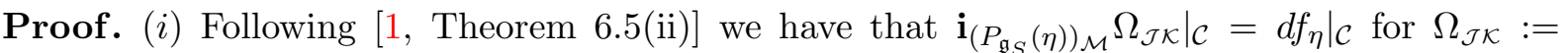
$\Omega_{\mathcal{M}}+\left\langle\mathcal{J}, \mathcal{K}_{\mathcal{W}}\right\rangle$. Then for each $\eta \in \mathfrak{g}$,

$$
\begin{aligned}
X_{\mathrm{nh}}\left(f_{\eta}\right) & =d f_{\eta}\left(X_{\mathrm{nh}}\right)=\Omega_{\mathcal{J K}}\left(\left(P_{\mathfrak{g}_{S}}(\eta)\right)_{\mathcal{M}}, X_{\mathrm{nh}}\right) \\
& =\Omega_{\mathcal{M}}\left(\left(P_{\mathfrak{g}_{S}}(\eta)\right)_{\mathcal{M}}, X_{\mathrm{nh}}\right)+\left\langle\mathcal{J}, \mathcal{K}_{\mathcal{W}}\right\rangle\left(\left(P_{\mathfrak{g}_{S}}(\eta)\right)_{\mathcal{M}}, X_{\mathrm{nh}}\right) .
\end{aligned}
$$

Since $\left\langle\mathcal{J}, \mathcal{K}_{\mathcal{W}}\right\rangle\left(X_{\mathrm{nh}},\left(P_{\mathfrak{g}_{S}}(\eta)\right)_{\mathcal{M}}\right)=0$ then, by the $G$-invariance of $\mathcal{H}_{\mathcal{M}}$,

$$
X_{\mathrm{nh}}\left(f_{\eta}\right)=\Omega_{\mathcal{M}}\left(\left(P_{\mathfrak{g}_{S}}(\eta)\right)_{\mathcal{M}}, X_{\mathrm{nh}}\right)=-d \mathcal{H}_{\mathcal{M}}\left(\left(P_{\mathfrak{g}_{S}}(\eta)\right)_{\mathcal{M}}\right)=0 .
$$

Assertion (ii) is just a consequence of Proposition 3.3(ii), since $P_{\mathfrak{g}_{S}}(\eta) \in \Gamma\left(\mathfrak{g}_{S}\right)$ and it is a generator of the first integral $f_{\eta}$.

In other words, if $W$ satisfies the vertical symmetry condition and

$$
\left\langle\mathcal{J}, \mathcal{K}_{\mathcal{W}}\right\rangle\left(X_{\mathrm{nh}},\left(P_{\mathfrak{g}_{S}}(\eta)\right)_{\mathcal{M}}\right)=0
$$

then, for $\eta \in \mathfrak{g}, P_{\mathfrak{g}_{S}}(\eta) \in \Gamma\left(\mathfrak{g}_{S}\right)$ is a horizontal gauge symmetry and $f_{\eta}$ is the associated horizontal gauge momentum.

Remark 3.9. When $W$ satisfies the vertical symmetry condition, there is a Lie subgroup $G_{\mathcal{W}}$ of $G$ integrating the Lie algebra $\mathfrak{g}_{W}$ and the nonholonomic system is $G_{\mathcal{W}^{-}}$Chaplygin, see [2]. The reduction of the nonholonomic system (on the Lagrangian side) by the Lie group $G_{\mathcal{W}}$ gives the Lagrange d'Alembert Poincaré equations on $T\left(Q / G_{\mathcal{W}}\right)$. However there is a remaining Lie group denoted by $H=G / G_{\mathcal{W}}$ that is a symmetry of the equations on $T\left(Q / G_{\mathcal{W}}\right)$. The coordinate version of condition (3.8) appears already in [35, Theorem 6] but written on $T\left(Q / G_{\mathcal{W}}\right)$, after performing the reduction by $G_{\mathcal{W}}$.

Proposition 3.10. Consider a nonholonomic system $(Q, L, D)$ with a $G$-symmetry that satisfies the dimension assumption. If the vertical complement $W$ satisfies the vertical symmetry condition and also if $\left\langle\mathcal{J}, \mathcal{K}_{\mathcal{W}}\right\rangle\left(X_{\mathrm{nh}}, \eta_{\mathcal{M}}\right)=0$ for all $\eta \in \mathfrak{g}$, then there are $k:=\operatorname{rank} S$ linearly independent horizontal gauge momenta of the form

$$
f_{\eta}=\left\langle\mathcal{J}^{\mathrm{nh}}, P_{\mathfrak{g}_{S}}(\eta)\right\rangle,
$$

for $\eta \in \mathfrak{g}$. 
Proof. By the vertical symmetry condition, let us consider a basis of constant sections $\left\{\tilde{\eta}_{1}, \ldots\right.$, $\left.\tilde{\eta}_{n}\right\}$ of $\mathfrak{g}_{W}$. Complete now the basis so that $\mathfrak{B}=\left\{\eta_{1}, \ldots, \eta_{k}, \tilde{\eta}_{1}, \ldots, \tilde{\eta}_{n}\right\}$ is a basis of $\mathfrak{g}$ (i.e., $\eta_{i}$ are constant sections of $\left.\mathfrak{g} \times Q \rightarrow Q\right)$. Let us define $f_{i}=\left\langle\mathcal{J}^{\mathrm{nh}}, P_{\mathfrak{g}_{S}}\left(\eta_{i}\right)\right\rangle, i=1, \ldots, k$. We claim that $f_{i}, i=1, \ldots, k$ are $k$ functionally independent first integrals of the nonholonomic dynamics. Since $\left\langle\mathcal{J}, \mathcal{K}_{\mathcal{W}}\right\rangle\left(X_{\mathrm{nh}}, P_{\mathfrak{g}_{S}}\left(\eta_{i}\right)\right\rangle=0, i=1, \ldots, k$, the functions $f_{1}, \ldots, f_{k}$ are constants along the motions by Proposition 3.8. To see that they are functionally independent, we need to show that the function $F=\left(f_{1}, \ldots, f_{k}\right): \mathcal{M} \longrightarrow \mathbb{R}^{k}$ is a submersion. Now suppose that $c_{i} d\left\langle\mathcal{J}^{\mathrm{nh}}, P_{\mathfrak{g}_{S}}\left(\eta_{i}\right)\right\rangle=0$ on $\mathcal{M}$ for $\left(c_{1}, \ldots, c_{k}\right) \in \mathbb{R}^{k}$ and $\left(c_{1}, \ldots, c_{k}\right) \neq(0, \ldots, 0)$ (or at least that $c_{i} d\left\langle\mathcal{J}^{\mathrm{nh}}, P_{\mathfrak{g}_{S}}\left(\eta_{i}\right)\right\rangle=0$ on an open set $\left.U \subset \mathcal{M}\right)$. In particular, $\left.d\left\langle\mathcal{J}^{\mathrm{nh}}, P_{\mathfrak{g}_{S}}(\eta)\right\rangle\right|_{\mathcal{c}}=0$ for $\eta=c_{i} \eta_{i}$. On the other hand, again using [1, Theorem 6.5(ii)] (as in proof of Proposition 3.8) we have that $\left.\mathbf{i}_{\left(P_{\mathfrak{g}_{S}}(\eta)\right)_{\mathcal{M}}} \Omega_{\mathcal{J K}}\right|_{\mathcal{C}}=d\left\langle\mathcal{J}^{\text {nh }}, P_{\mathfrak{g}_{S}}(\eta)\right\rangle_{\mathcal{C}}=0$ for $\Omega_{\mathcal{J K}}:=\Omega_{\mathcal{M}}+\left\langle\mathcal{J}, \mathcal{K}_{\mathcal{W}}\right\rangle$. Since $\left.\Omega_{\mathcal{J K}}\right|_{\mathcal{C}}$ is nondegenerate $\left(\left[1\right.\right.$, Section 5.1] or $\left[3\right.$, Proposition 3]), then $\left(P_{\mathfrak{g}_{S}}(\eta)\right)_{\mathcal{M}}=0$ which implies that $\eta=c_{i} \eta_{i} \in \mathfrak{g}_{W}$ that is in contradiction with the choice of our basis.

\section{Examples}

\subsection{Vertical rolling disk}

We consider a (homogeneous) disk which is constrained to roll without sliding on a horizontal plane while standing vertically $[9,18,30,32]$. The configuration manifold is $Q=\mathbb{R}^{2} \times S^{1} \times S^{1}$ with coordinates $(x, y, \varphi, \psi)$, where $(x, y) \in \mathbb{R}^{2}$ are Cartesian coordinates of the contact point, $\psi$ is the angle between the $x$-axis and the projection of the disk on the plane and $\varphi$ is the angle between a fixed radius of the disk and the vertical. Assuming that the disk has unit mass the Lagrangian is

$$
L=\frac{1}{2}\left(\dot{x}^{2}+\dot{y}^{2}\right)+\frac{1}{2} I \dot{\varphi}^{2}+\frac{1}{2} J \dot{\psi}^{2},
$$

where $I$ and $J$ are the pertinent moments of inertia. The nonholonomic constraints describing the rolling motion without slipping are given by

$$
\dot{x}=R \cos \psi \dot{\varphi}, \quad \dot{y}=R \sin \psi \dot{\varphi},
$$

and they define the constraint distribution $D$ on $Q$ with fibers

$$
D_{(x, y, \varphi, \psi)}=\operatorname{span}\left\{R \cos \psi \partial_{x}+R \sin \psi \partial_{y}+\partial_{\varphi}, \partial_{\psi}\right\}
$$

The group $\mathrm{SE}(2) \times S^{1}$ is a symmetry of the system with respect to the action on $Q$ given by

$$
((a, b, \lambda), \mu) \cdot(x, y, \varphi, \psi)=(a+x \cos \lambda-y \sin \lambda, b+x \sin \lambda+y \cos \lambda, \varphi+\lambda, \psi+\mu)
$$

for $(a, b, \lambda) \in \mathrm{SE}(2)$ and $\mu \in S^{1}$.

Let us now pass to the Hamiltonian formulation on the cotangent bundle. In canonical coordinates $\left(x, y, \varphi, \psi ; p_{x}, p_{y}, p_{\varphi}, p_{\psi}\right)$ on $T^{*} Q$, the constraint submanifold $\mathcal{M}$ is given by

$$
\mathcal{M}=\left\{\left(x, y, \varphi, \psi, p_{x}, p_{y}, p_{\varphi}, p_{\psi}\right): p_{x}=\frac{R}{I} p_{\varphi} \cos \psi \text { and } p_{y}=\frac{R}{I} p_{\varphi} \sin \psi\right\}
$$

and since the Hamiltonian on $T^{*} Q$ is $\mathcal{H}=\frac{1}{2}\left(p_{x}^{2}+p_{y}^{2}\right)+\frac{1}{2 I} p_{\varphi}^{2}+\frac{1}{2 J} p_{\psi}^{2}$, then $\mathcal{H}_{\mathcal{M}}$ reads $\mathcal{H}_{\mathcal{M}}=$ $\frac{1}{2 I}\left(\frac{R^{2}}{I}+1\right) p_{\varphi}^{2}+\frac{1}{2 J} p_{\psi}^{2}$.

Observe that the vertical distribution $V$ has fibers $V_{(x, y, \varphi, \psi)}=\operatorname{span}\left\{\partial_{x}, \partial_{y}, \partial_{\varphi}, \partial_{\psi}\right\}$, and that $S=V \cap D=D$. Therefore the vertical complement $W$ can be chosen to be $W=\operatorname{span}\left\{\partial_{x}, \partial_{y}\right\}$ 
and we can check that it satisfies the vertical symmetry condition, Definition 3.7. Moreover $\left\langle\mathcal{J}, \mathcal{K}_{\mathcal{W}}\right\rangle=0$ (see [1]), since

$$
\mathcal{J}=\left(\frac{R}{I} p_{\varphi} \cos \psi, \frac{R}{I} p_{\varphi} \sin \psi, p_{\varphi}, p_{\psi}\right) \in \mathfrak{g}^{*}
$$

and the $\mathcal{W}$-curvature is $\mathcal{K}_{\mathcal{W}}=(\sin \psi d \psi \wedge d \varphi,-\cos \psi d \psi \wedge d \varphi, 0,0)$. Thus the hypotheses of Propositions 3.8 and 3.10 are satisfied and then the system admits two independent horizontal gauge momenta. Since

$$
\mathfrak{g}_{S}=\operatorname{span}\{(R \cos \psi, R \sin \psi, 1,0),(-y, x, 0,1)\}, \quad \mathfrak{g}_{W}=\operatorname{span}\{(1,0,0,0),(0,1,0,0)\},
$$

then

$$
\xi_{1}:=P_{\mathfrak{g}_{S}}((0,0,1,0))=(R \cos \psi, R \sin \psi, 1,0), \quad \xi_{2}:=P_{\mathfrak{g}_{S}}((0,0,0,1))=(-y, x, 0,1),
$$

are horizontal gauge symmetries. In fact, the associated $\mathcal{M}$-cotangent lifts are $\left(\xi_{1}\right)_{Q}^{\mathcal{M}}=\left(\xi_{1}\right)_{\mathcal{M}}=$ $R \cos \psi \partial_{x}+R \sin \psi \partial_{y}+\partial_{\varphi}$ and $\left(\xi_{2}\right)_{Q}^{\mathcal{M}}=\left(\xi_{2}\right)_{\mathcal{M}}=\partial_{\psi}$ and thus $\left(\xi_{1}\right)_{Q}^{\mathcal{M}}\left(\mathcal{H}_{\mathcal{M}}\right)=\left(\xi_{2}\right)_{Q}^{\mathcal{M}}\left(\mathcal{H}_{\mathcal{M}}\right)=0$. Therefore $\mathcal{J}_{1}=\left\langle\mathcal{J}^{\text {nh }}, \xi_{1}\right\rangle=\left(\frac{R^{2}}{I}+1\right) p_{\varphi}$ and $\mathcal{J}_{2}=\left\langle\mathcal{J}^{\text {nh }}, \xi_{2}\right\rangle=p_{\psi}$ are horizontal gauge momenta.

\subsection{The ball rolling on a plane}

Consider the classical example of a inhomogeneous ball of radius $r$ whose center of mass coincides with the geometric center of the ball that rolls without sliding on a plane [10, 12]. We will follow the notation and viewpoint of $[1,23]$. The configuration manifold is $Q=\mathrm{SO}(3) \times \mathbb{R}^{2}$ where $g \in \mathrm{SO}(3)$ represents the orientation of the ball and $(x, y) \in \mathbb{R}^{2}$ the point of contact of the ball with the plane. Consider the (left invariant) moving frame $\left\{X_{1}^{L}, X_{2}^{L}, X_{3}^{L}\right\}$ on $T_{g}(\mathrm{SO}(3))$ given by left translations of the canonical basis $\left\{e_{1}, e_{2}, e_{3}\right\}$ of $\mathfrak{s o}(3) \simeq \mathbb{R}^{3}$ and denote by $\boldsymbol{\Omega}=\left(\Omega_{1}, \Omega_{2}, \Omega_{2}\right)$ the angular velocities of the body with respect to this moving frame. Then, the non-sliding constraints are given by

$$
\dot{x}=r\langle\boldsymbol{\beta}, \boldsymbol{\Omega}\rangle \quad \text { and } \quad \dot{y}=-r\langle\boldsymbol{\alpha}, \boldsymbol{\Omega}\rangle,
$$

where $\boldsymbol{\alpha}$ and $\boldsymbol{\beta}$ are the first and second rows of the matrix $g \in \mathrm{SO}(3)$ and $\langle\cdot, \cdot\rangle$ is the canonical pairing in $\mathbb{R}^{3}$. The non-integrable distribution $D$ is then generated by

$$
\begin{aligned}
& \left\{X_{1}:=X_{1}^{L}+r \beta_{1} \frac{\partial}{\partial x}-r \alpha_{1} \frac{\partial}{\partial y}, X_{2}:=X_{2}^{L}+r \beta_{2} \frac{\partial}{\partial x}-r \alpha_{2} \frac{\partial}{\partial y},\right. \\
& \left.X_{3}:=X_{3}^{L}+r \beta_{3} \frac{\partial}{\partial x}-r \alpha_{3} \frac{\partial}{\partial y}\right\} .
\end{aligned}
$$

Let us consider the action of the Lie group $G=\left\{(h, a) \in \mathrm{SO}(3) \times \mathbb{R}^{2}: h \cdot e_{3}=e_{3}\right\}$ on $Q$ given by

$$
(h, a):(g,(x, y)) \mapsto\left(h g,(x, y) \tilde{h}^{t}+a\right) \in Q,
$$

where $\tilde{h}$ is the $2 \times 2$ rotational matrix determined by $h$. This action is a symmetry of the nonholonomic system since it leaves invariant the distribution $D$ and the lagrangian $L(g, x, y ; \Omega, \dot{x}, \dot{y})=$ $\frac{1}{2}\left(\langle\mathbb{I} \boldsymbol{\Omega}, \boldsymbol{\Omega}\rangle+m\left(\dot{x}^{2}+\dot{y}^{2}\right)\right)$, where $\mathbb{I}$ the inertia tensor (II is represented by a diagonal matrix with entries $\left.\mathbb{I}_{1}, \mathbb{I}_{2}, \mathbb{I}_{3}\right)$. Note that the Lie algebra $\mathfrak{g}$ associated to $G$ is $\mathbb{R} \times \mathbb{R}^{2}$ with the trivial structure and also

$$
(1 ; 0,0)_{Q}=\langle\boldsymbol{\gamma}, \mathbf{X}\rangle-y \frac{\partial}{\partial x}+x \frac{\partial}{\partial y}, \quad(0 ; 1,0)_{Q}=\frac{\partial}{\partial x} \quad \text { and } \quad(0 ; 0,1)_{Q}=\frac{\partial}{\partial y},
$$


where $\gamma=\left(\gamma_{1}, \gamma_{2}, \gamma_{3}\right)$ is the third row of the rotational matrix $g$ and $\mathbf{X}=\left(X_{1}, X_{2}, X_{3}\right)$. Therefore, we can choose the vertical complement $W$ to be $W=\operatorname{span}\left\{Z_{1}:=\partial_{x}, Z_{2}:=\partial_{y}\right\}$ and then

$$
\mathfrak{g}_{S}=\operatorname{span}\{(1 ; y,-x)\} \quad \text { and } \quad \mathfrak{g}_{W}=\operatorname{span}\{(0 ; 0,1),(0 ; 1,0)\} .
$$

Now we will define the submanifold $\mathcal{M} \subset T^{*} Q$. Let us denote by $\boldsymbol{\lambda}=\left(\lambda_{1}, \lambda_{2}, \lambda_{3}\right)$ where $\left\{\lambda_{1}, \lambda_{2}, \lambda_{3}\right\}$ is the (local) basis of $T^{*}(\mathrm{SO}(3))$ that is dual to the moving frame $\left\{X_{1}^{L}, X_{2}^{L}, X_{3}^{L}\right\}$. Following $[1,23]$ we consider the basis $\left\{\lambda_{1}, \lambda_{2}, \lambda_{3}, \epsilon_{x}:=d x-r\langle\boldsymbol{\beta}, \boldsymbol{\lambda}\rangle, \epsilon_{y}:=d y+r\langle\boldsymbol{\alpha}, \boldsymbol{\lambda}\rangle\right\}$ of $T^{*} Q$ that is dual to the adapted basis $\left\{X_{1}, X_{2}, X_{3}, Z_{1}, Z_{2}\right\}$ of $T Q=D \oplus W$. Then if $\left(K_{1}, K_{2}, K_{3}, \tilde{p}_{x}, \tilde{p}_{y}\right)$ are the coordinates on $T^{*} Q$ associated to the dual basis, the submanifold $\mathcal{M}$ is given by

$$
\begin{aligned}
\mathcal{M}=\left\{\left(g, x, y ; K_{1}, K_{2}, K_{3}, \tilde{p}_{x}, \tilde{p}_{y}\right):\right. & \mathbf{K}=\mathbb{I} \boldsymbol{\Omega}+m r^{2}(\boldsymbol{\Omega}-\langle\boldsymbol{\gamma}, \boldsymbol{\Omega}\rangle \boldsymbol{\gamma}), \\
\tilde{p}_{x} & \left.=m r\langle\boldsymbol{\beta}, \boldsymbol{\Omega}\rangle \text { and } \tilde{p}_{y}=-m r\langle\boldsymbol{\alpha}, \boldsymbol{\Omega}\rangle\right\},
\end{aligned}
$$

where $\mathbf{K}=\left(K_{1}, K_{2}, K_{3}\right)$.

An arbitrary section $\xi \in \Gamma\left(\mathfrak{g}_{S}\right)$ has the form

$$
\xi=\phi(g,(x, y))(1 ; y,-x)=\phi \cdot(1 ; 0,0)+y \phi \cdot(0 ; 1,0)-y \phi .(0 ; 0,1)
$$

for $\phi \in C^{\infty}(Q)$ and also we where we denote $\phi=\phi(g,(x, y))$. Then from Lemma 3.2 the $\mathcal{M}$-cotangent lift of $\xi$ is

$$
\begin{aligned}
\xi_{Q}^{\mathcal{M}}= & \phi\langle\gamma, \mathbf{X}\rangle+(\langle\boldsymbol{\gamma}, \mathbf{K}\rangle-m r(y\langle\boldsymbol{\beta}, \boldsymbol{\Omega}\rangle+x\langle\boldsymbol{\alpha}, \boldsymbol{\Omega}\rangle)) \pi_{\mathrm{nh}}^{\sharp}(d \phi) \\
& +m r\left(\langle\boldsymbol{\beta}, \boldsymbol{\Omega}\rangle \pi_{\mathrm{nh}}^{\sharp}(d(y \phi))+\langle\boldsymbol{\alpha}, \boldsymbol{\Omega}\rangle \pi_{\mathrm{nh}}^{\sharp}(d(x \phi))\right),
\end{aligned}
$$

where we used that $\Theta_{\mathcal{M}}=\langle\mathbf{K}, \boldsymbol{\lambda}\rangle+\iota^{*}\left(\tilde{p}_{x}\right) \epsilon_{x}+\iota^{*}\left(\tilde{p}_{y}\right) \epsilon_{y}$ to compute $\left\langle\mathcal{J}, \eta_{i}\right\rangle$. Knowing that the restricted hamiltonian $\mathcal{H}_{\mathcal{M}}: \mathcal{M} \rightarrow \mathbb{R}$ is $\mathcal{H}_{\mathcal{M}}=\frac{1}{2}\langle\mathbf{K}, \boldsymbol{\Omega}\rangle$, following Proposition 3.3(ii) we should look for a function $\phi \in C^{\infty}(Q)$ such that

$$
\begin{aligned}
& d \mathcal{H}_{\mathcal{M}}\left((\langle\boldsymbol{\gamma}, \mathbf{K}\rangle-m r\langle y \boldsymbol{\beta}+x \boldsymbol{\alpha}, \boldsymbol{\Omega}\rangle) \pi_{\mathrm{nh}}^{\sharp}(d \phi)\right. \\
& \left.\quad+m r\left(\langle\boldsymbol{\beta}, \boldsymbol{\Omega}\rangle \pi_{\mathrm{nh}}^{\sharp}(d(y \phi))+\langle\boldsymbol{\alpha}, \boldsymbol{\Omega}\rangle \pi_{\mathrm{nh}}^{\sharp}(d(x \phi))\right)\right)=0
\end{aligned}
$$

to guarantee that $\xi$ is a horizontal gauge symmetry and so $\mathcal{J}_{\xi}=\left\langle\mathcal{J}^{\text {nh }}, \xi\right\rangle$ is a first integral. However, we can check that the vertical complement $W$ satisfies the vertical symmetry condition (see Definition 3.7) and therefore we have a natural candidate for the horizontal gauge symmetry: from Proposition $3.8, \xi=P_{\mathfrak{g}_{S}}(1 ; 0,0)=(1 ; y,-x)$ is a horizontal gauge symmetry if $\left\langle\mathcal{J}, \mathcal{K}_{\mathcal{W}}\right\rangle\left(X_{\mathrm{nh}}, \xi_{\mathcal{M}}\right)=0$ where, in this case, $\xi_{\mathcal{M}}=\langle\boldsymbol{\gamma}, \mathbf{X}\rangle$. From [1] we have that

$$
\left\langle\mathcal{J}, \mathcal{K}_{\mathcal{W}}\right\rangle=m r^{2}\langle\boldsymbol{\Omega}-\langle\boldsymbol{\gamma}, \boldsymbol{\Omega}\rangle \boldsymbol{\gamma}, d \boldsymbol{\lambda}\rangle
$$

where $d \boldsymbol{\lambda}=\left(\lambda_{2} \wedge \lambda_{3}, \lambda_{3} \wedge \lambda_{1}, \lambda_{1} \wedge \lambda_{2}\right)$. It is easy to check that $\left\langle\mathcal{J}, \mathcal{K}_{\mathcal{W}}\right\rangle\left(X_{\mathrm{nh}},\langle\boldsymbol{\gamma}, \mathbf{X}\rangle\right)=0$ for $X_{\mathrm{nh}}=\langle\boldsymbol{\Omega}, \mathbf{X}\rangle+\left\langle\mathbf{K} \times \boldsymbol{\Omega}, \partial_{\mathbf{K}}\right\rangle$. Therefore the section $\xi=P_{\mathfrak{g}_{S}}(1 ; 0,0)=(1 ; y,-x)$ is the horizontal gauge symmetry and $\mathcal{J}_{\xi}=\mathbf{i}_{\langle\gamma, \mathbf{X}\rangle} \Theta_{\mathcal{M}}=\langle\gamma, \mathbf{K}\rangle$ is a first integral of the dynamics, i.e., $\mathcal{J}_{\xi}$ is a horizontal gauge symmetry.

From Proposition 3.10 we observe that $\operatorname{rank} S=1$ and so $\mathcal{J}_{\xi}$ is the only (up to constants) horizontal gauge symmetries of the form (3.9).

Remark 4.1. Observe that $£_{\xi_{\mathcal{M}}} \Omega_{\mathcal{M}}=d \mathcal{J}_{\xi}-m r\langle\boldsymbol{\Omega}, d \gamma\rangle+\Sigma$ where $\Sigma$ satisfies that $\Sigma(X)=0$ for all $X \in \Gamma(\mathcal{C})$. However, it is easy to check that $\left.m r\langle\boldsymbol{\Omega}, d \boldsymbol{\gamma}\rangle\right|_{\mathcal{C}} \neq 0$ and therefore, the vector field $\xi_{\mathcal{M}}=\langle\gamma, \mathbf{X}\rangle$ is not a Cartan symmetry in the sense of [15], see Remark 3.4. 


\section{Conclusions and perspectives}

The main goal of this work is to show how certain first integrals linear in the momenta of a nonholonomic systems with a $G$-symmetry might be generated by the symmetry group. In particular, in Definition 3.1 and Corollary 3.5 we define the $\mathcal{M}$-cotangent lift and we use it to characterize horizontal gauge symmetries. Then, under the dimension assumption (3.3), the tangent bundle can be splitted (see (3.4)) in the direct sum of the constraint distribution $D$ and a vertical complement $W$. If $W$ satisfies the so-called vertical symmetry condition (Definition 3.7), Proposition 3.8 guarantees that the nonholonomic system admits horizontal gauge momenta induced by elements of the Lie algebra, while Proposition 3.10 tells that the number of (linearly independent) horizontal gauge momenta is given by the rank of the distribution $S=D \cap V$.

It is important to note that the choice of the vertical complement $W$ is not unique. Usually, the most natural choice for $W$ is a complement that satisfies the vertical symmetry condition, as we did in Examples 4.1 and 4.2. This condition is a 'natural' condition that arises in the framework of reduction and Hamiltonization, see [1]. However, in some cases choosing a complement that satisfies the vertical symmetry condition might be too strong to study a group origin of first integrals (linear in the momenta). The cases where we need a vertical complement $W$ that does not satisfy the vertical symmetry condition is our topic of future work and investigation.

More precisely, in the following example we show that when we choose a complement $W$ satisfying the vertical symmetry condition then, for $\eta \in \mathfrak{g},\left\langle\mathcal{J}, \mathcal{K}_{\mathcal{W}}\right\rangle\left(X_{\mathrm{nh}},\left(P_{\mathfrak{g}_{S}}(\eta)\right)_{\mathcal{M}}\right) \neq 0$ (cf. Proposition 3.8). However, the choice of a different vertical complement $W$ allows us to write the horizontal gauge symmetry in terms of a section $P_{\mathfrak{g}_{S}}(\eta) \in \Gamma\left(\mathfrak{g}_{S}\right)$ for $\eta \in \mathfrak{g}$; but in this case the vertical complement $W$ does not verify the vertical symmetry condition.

Example 5.1 (the nonholonomic particle [5, 32]). The classical example of the nonholonomic particle describes a particle in $\mathbb{R}^{3}$ subjected to the nonholonomic constraints $\dot{z}=y \dot{x}$ and where the Lagrangian is the canonical kinetic energy metric on $\mathbb{R}^{3}$. Then, in canonical coordinates the distribution $D$ has fibers $D_{(x, y, z)}=\operatorname{span}\left\{\partial_{y}, \partial_{x}+y \partial z\right\}$, the constraint manifold is

$$
\mathcal{M}=\left\{\left(x, y, z ; p_{x}, p_{y}, p_{z}\right): p_{z}=y p_{x}\right\}
$$

and the restricted Hamiltonian is $\mathcal{H}_{\mathcal{M}}=\frac{1}{2}\left(\left(1+y^{2}\right) p_{x}^{2}+p_{y}^{2}\right)$. The nonholonomic vector field is

$$
X_{\mathrm{nh}}=p_{x}\left(\frac{\partial}{\partial x}+y \frac{\partial}{\partial z}\right)+p_{y} \frac{\partial}{\partial y}-\frac{y p_{x} p_{y}}{1+y^{2}} \frac{\partial}{\partial p_{x}} .
$$

It is well known that the function $f\left(x, y, z, p_{x}, p_{y}\right)=p_{x} \sqrt{1+y^{2}}$ is a first integral of the system and that it is a horizontal gauge momentum, with respect to the $G$-symmetry given by the translational action of $\mathbb{R}^{2}$ on $\mathbb{R}^{3}$. The vertical space associated to the $G$-action is $V=$ $\operatorname{span}\left\{\partial_{x}, \partial_{z}\right\}$, and the subbundle $\mathfrak{g}_{S}$ is locally generated by $\{(1, y)\}$. Then the function $f$ is a horizontal gauge momentum with horizontal gauge symmetry $\xi=\sqrt{1+y^{2}}(1, y) \in \Gamma\left(\mathfrak{g}_{S}\right)$, i.e., $\xi_{Q}^{T^{*} Q}(\mathcal{H})(m)=0$ for $m \in \mathcal{M}$ and thus $f=\mathcal{J}_{\xi}$ (see [18]).

Now we are going to give a geometric interpretation of $\xi$ in terms of the tools of Section 3 , that is, by choosing a vertical complement of the constraint distribution. An obvious choice for the $G$-invariant vertical complement $W$ so that it satisfies the vertical symmetry condition is $W=\operatorname{span}\left\{\partial_{z}\right\} .^{5}$ Observe that $\mathfrak{g}_{W}=\operatorname{span}\{(0,1)\}$. It is straightforward to check that for any $(a, b) \in \mathfrak{g}=\mathbb{R}^{2}, P_{\mathfrak{g}_{S}}((a, b))=a(1, y)$, so there is no element $\eta \in \mathfrak{g}$ such that $P_{\mathfrak{g}_{S}}(\eta)$ gives the horizontal gauge symmetry $\xi=\sqrt{1+y^{2}}(1, y)$. Indeed, using that $\left\langle\mathcal{J}, \mathcal{K}_{\mathcal{W}}\right\rangle=y p_{x} d x \wedge d y$, we check that $\left\langle\mathcal{J}, \mathcal{K}_{\mathcal{W}}\right\rangle\left(X_{\mathrm{nh}},\left(P_{\mathfrak{g}_{S}}((1,0))\right)_{\mathcal{M}}\right) \neq 0$ (see [1]).

\footnotetext{
${ }^{5}$ This is the choice done in [1] in the framework of Hamiltonization.
} 
However, it is possible to change $W$ in order to obtain a horizontal gauge symmetry and a horizontal gauge momentum of the form $P_{\mathfrak{g}_{S}}(\eta) \in \Gamma\left(\mathfrak{g}_{S}\right)$ and $f_{\eta}=\left\langle\mathcal{J}^{\text {nh }}, P_{\mathfrak{g}_{S}}(\eta)\right\rangle$.

Consider the $G$-invariant vertical complement $W$ whose fibers are given by

$$
W_{(x, y, z)}=\operatorname{span}\left\{\left(1-\sqrt{1+y^{2}}\right) \frac{\partial}{\partial x}+y \frac{\partial}{\partial z}\right\} .
$$

The splitting (3.7) of the Lie algebra $\mathfrak{g}$ is given by

$$
\mathfrak{g}_{S}=\operatorname{span}\{(1, y)\} \quad \text { and } \quad \mathfrak{g}_{W}=\operatorname{span}\left\{\left(1-\sqrt{1+y^{2}}, y\right)\right\} .
$$

Observe that $W$ does not satisfy the vertical symmetry condition, however

$$
P_{\mathfrak{g}_{S}}((1,0))=\sqrt{1+y^{2}}(1, y)=\xi
$$

where $P_{\mathfrak{g}_{S}}: \mathbb{R}^{2} \rightarrow \mathfrak{g}_{S}$ is the projection associated to the splitting defined in (5.2). Observe that the projection $P_{\mathfrak{g}_{S}}$ is induced by the choice of the vertical complement $W$ given in (5.1). In this case, the horizontal gauge momentum $f$ is exactly $f=\left\langle\mathcal{J}^{\mathrm{nh}}, P_{\mathfrak{g}_{S}}((1,0))\right\rangle$.

It is then clear that a deeper study of this topic is necessary as a more geometric understanding of the Noetherian character (see $[18,19]$ ) of horizontal gauge momenta (see [4]).

\section{Acknowledgments}

This work is partially supported by the research projects Symmetries and integrability of nonholonomic mechanical systems of the University of Padova. N.S. wishes to thank IMPA and H. Bursztyn for the kind hospitality during which this work took origin. P.B. thanks the financial support of CAPES (grants PVE 11/2012 and PVE 089/2013) and CNPq's Universal grant. We also thank the anonymous referees for their useful comments.

\section{References}

[1] Balseiro P., The Jacobiator of nonholonomic systems and the geometry of reduced nonholonomic brackets, Arch. Ration. Mech. Anal. 214 (2014), 453-501, arXiv:1301.1091.

[2] Balseiro P., Fernandez O.E., Reduction of nonholonomic systems in two stages and Hamiltonization, Nonlinearity 28 (2015), 2873-2912, arXiv:1409.0456.

[3] Balseiro P., García-Naranjo L.C., Gauge transformations, twisted Poisson brackets and Hamiltonization of nonholonomic systems, Arch. Ration. Mech. Anal. 205 (2012), 267-310, arXiv:1104.0880.

[4] Balseiro P., Sansonetto N., Conserved quantities and Hamiltonization, work in progress.

[5] Bates L., Graumann H., MacDonnell C., Examples of gauge conservation laws in nonholonomic systems, Rep. Math. Phys. 37 (1996), 295-308.

[6] Bates L., Śniatycki J., Nonholonomic reduction, Rep. Math. Phys. 32 (1993), 99-115.

[7] Benenti S., Meccanica dei sistemi anolonomi, in Complementi alle Lezioni di Meccanica Razionale di T. LeviCivita e U. Amaldi, Editors E.N.M. Cirillo, G. Maschio, T. Ruggeri, G. Saccomandi, CompoMat, 2012, 213-257.

[8] Bloch A.M., Nonholonomic mechanics and control, Interdisciplinary Applied Mathematics, Vol. 24, SpringerVerlag, New York, 2003.

[9] Bloch A.M., Krishnaprasad P.S., Marsden J.E., Murray R.M., Nonholonomic mechanical systems with symmetry, Arch. Rational Mech. Anal. 136 (1996), 21-99.

[10] Borisov A.V., Mamaev I.S., Chaplygin's ball rolling problem is Hamiltonian, Math. Notes 70 (2001), $720-$ 723.

[11] Borisov A.V., Mamaev I.S., Conservation laws, hierarchy of dynamics and explicit integration of nonholonomic systems, Regul. Chaotic Dyn. 13 (2008), 443-490. 
[12] Borisov A.V., Mamaev I.S., Kilin A.A., Rolling of a ball on a surface. New integrals and hierarchy of dynamics, Regul. Chaotic Dyn. 7 (2002), 201-219, nlin.SI/0303024.

[13] Cantrijn F., de León M., Marrero J.C., de Diego D.M., Reduction of nonholonomic mechanical systems with symmetries, Rep. Math. Phys. 42 (1998), 25-45.

[14] Cortés Monforte J., Geometric, control and numerical aspects of nonholonomic systems, Lecture Notes in Math., Vol. 1793, Springer-Verlag, Berlin, 2002.

[15] Crampin M., Mestdag T., The Cartan form for constrained Lagrangian systems and the nonholonomic Noether theorem, Int. J. Geom. Methods Mod. Phys. 8 (2011), 897-923, arXiv:1101.3153.

[16] Crampin M., Pirani F.A.E., Applicable differential geometry, London Mathematical Society Lecture Note Series, Vol. 59, Cambridge University Press, Cambridge, 1986.

[17] Cushman R., Duistermaat H., Śniatycki J., Geometry of nonholonomically constrained systems, Advanced Series in Nonlinear Dynamics, Vol. 26, World Scientific Publishing Co. Pte. Ltd., Hackensack, NJ, 2010.

[18] Fassò F., Giacobbe A., Sansonetto N., Gauge conservation laws and the momentum equation in nonholonomic mechanics, Rep. Math. Phys. 62 (2008), 345-367.

[19] Fassò F., Giacobbe A., Sansonetto N., On the number of weakly Noetherian constants of motion of nonholonomic systems, J. Geom. Mech. 1 (2009), 389-416.

[20] Fassò F., Giacobbe A., Sansonetto N., Linear weakly Noetherian constants of motion are horizontal gauge momenta, J. Geom. Mech. 4 (2012), 129-136.

[21] Fassò F., Ramos A., Sansonetto N., The reaction-annihilator distribution and the nonholonomic Noether theorem for lifted actions, Regul. Chaotic Dyn. 12 (2007), 579-588.

[22] Fassò F., Sansonetto N., Conservation of 'moving' energy in nonholonomic systems with affine constraints and integrability of spheres on rotating surfaces, J. Nonlinear Sci., to appear, arXiv:1503.06661.

[23] García-Naranjo L., Reduction of almost Poisson brackets and Hamiltonization of the Chaplygin sphere, Discrete Contin. Dyn. Syst. Ser. S 3 (2010), 37-60, arXiv:0808.0854.

[24] Giachetta G., First integrals of non-holonomic systems and their generators, J. Phys. A: Math. Gen. 33 (2000), 5369-5389.

[25] Ibort A., de Leon M., Marrero J.C., Martin de Diego D., Dirac brackets in constrained dynamics, Fortschr. Phys. 47 (1999), 459-492.

[26] Jotz M., Ratiu T.S., Dirac structures, nonholonomic systems and reduction, Rep. Math. Phys. 69 (2012), 5-56, arXiv:0806.1261.

[27] Marle C.-M., Reduction of constrained mechanical systems and stability of relative equilibria, Comm. Math. Phys. 174 (1995), 295-318.

[28] Marle C.-M., Various approaches to conservative and nonconservative nonholonomic systems, Rep. Math. Phys. 42 (1998), 211-229.

[29] Marsden J.E., Ratiu T.S., Introduction to mechanics and symmetry. A basic exposition of classical mechanical systems, Texts in Applied Mathematics, Vol. 17, 2nd ed., Springer-Verlag, New York, 1999.

[30] Neimark Ju.I., Fufaev N.A., Dynamics of nonholonomic systems, Translations of Mathematical Monographs, Vol. 33, Amer. Math. Soc., Providence, RI, 1972.

[31] Ortega J.-P., Ratiu T.S., Momentum maps and Hamiltonian reduction, Progress in Mathematics, Vol. 222, Birkhäuser Boston, Inc., Boston, MA, 2004.

[32] Pars L.A., A treatise on analytical dynamics, Heinemann Educational Books Ltd., London, 1968.

[33] Śniatycki J., Nonholonomic Noether theorem and reduction of symmetries, Rep. Math. Phys. 42 (1998), $5-23$.

[34] van der Schaft A.J., Maschke B.M., On the Hamiltonian formulation of nonholonomic mechanical systems, Rep. Math. Phys. 34 (1994), 225-233.

[35] Zenkov D.V., Linear conservation laws of nonholonomic systems with symmetry, Discrete Contin. Dyn. Syst. (2003), suppl., 967-976. 